\title{
La etnografía institucional: alternativa metodológica en la investigación educativa*
}

\author{
Institutional ethnography: a methodological \\ alternative for educative research
}

Gabriel Vázquez Dzul**

\section{RESUMEN}

El uso de la etnografía en el análisis de la experiencia escolar provee de mayor claridad sobre la efectividad de las acciones institucionales dirigidas a la mejora educativa. Al momento su uso se ha limitado a la descripción detallada del comportamiento del estudiante dentro y fuera del aula. En respuesta este trabajo pretende otorgarle un uso integral con vistas a complementar los enfoques cuantitativos comunes en el área. Así, el propósito principal es bosquejar el uso de la etnografía institucional como una alternativa metodológica en la investigación educativa por medio del uso de varias herramientas e instrumentos cualitativos y cuantitativos.

Palabras clave: Etnografía, experiencia escolar, ambiente institucional

\begin{abstract}
Ethnography provides a clear-cut vision about the effectiveness of institutional actions for school improvement based on the school experience analysis. At this moment, the use of ethnography as a qualitative method has been reduced through the description of the student behavior inside and outside the classroom. As an alternative, this paper aims to give a holistic use of ethnography as a complement to quantitative approaches in educative research. Thus, the main purpose is to outline the use of institutional ethnography as a methodological alternative in educational research through the use of various qualitative and quantitative instruments.
\end{abstract}

Key words: Ethnography, school experience, institutional environment

\section{INTRODUCCIÓN}

Quienes nos dedicamos a la investigación educativa, por lo normal, realizamos estudios institucionales acudiendo a los estudiantes como objetos-sujetos de nuestro análisis con el propósito

\footnotetext{
"Agradezco a la Universidad de Quintana Roo, en México, por darme la oportunidad de desempeñarme durante casi tres años como Responsable del Área de Investigación Educativa del Departamento de Innovación Educativa. Externo agradecimientos a los Programas Federales que financiaron los proyectos de investigación que aquí se mencionan. Hago extensivo también el agradecimiento a todos los universitarios que colaboraron con éste y otros proyectos mientras estuve en el Área, en particular a Reynaldo Chí Aguilar y Rubí Caamal Solís.

"* Área de Sociales del Instituto Universitario Carl Rogers; gvazdzul@gmail.com.
} 
de generar información para atender problemas frecuentes de la educación. Así, la investigación aplicada a la mejora educativa es un lugar común en las acciones de los investigadores en educación o, al menos, esa es la encomienda fundamental. Ante este contexto primordial de la investigación educativa, cabe preguntarnos si la metodología actual permite la incubación de procesos reales de mejora en las líneas de estudio que nos planteemos. Al respecto, basta echar un vistazo al arsenal de textos elaborados con el propósito de identificar-por ejemplo- las causas de abandono escolar y, también, de discutir la terminología adecuada del proceso de ausencia en el aula, ${ }^{1}$ para encontrar puntos de quiebre del encuadre teórico-metodológico; esto es, la explicación tácita de factores que incidieron en la deserción sin considerar la dimensión institucional y social del proceso. Para ampliar el panorama, cabe decir que los alumnos que hace un par de años abandonaron sus estudios, sin duda, no lo hicieron por las mismas motivaciones que posiblemente tendrán los estudiantes actuales.

Decidí usar el ejemplo de la deserción (o abandono, ausentismo, etc.) como uno de los ejes problemáticos más comunes en las instituciones de educación superior, ya que sigue causando interés para diversos organismos nacionales e internacionales. Dicho esto, considero que quienes han seguido al pie de la letra metodologías preestablecidas en materia de investigación educativa, han derivado en obviedades que no contribuyen a los procesos de mejora ni realizan aportes que dirijan a la ruptura paradigmática en esta área de conocimiento. Una postura alternativa ante tal impasse teórico-metodológico es el maridaje de enfoques dirigido no solamente hacia la búsqueda de explicaciones causales, sino además hacia la reconstrucción de un sistema educativo conforme con la realidad social que le sustenta. Así, el propósito en este documento radica en la exploración de la etnografía como un método dirigido hacia la identificación de la congruencia entre la propuesta educativa institucional (modelo educativo y demás documentos rectores) y la experiencia estudiantil, tomando como

\footnotetext{
${ }^{1}$ Algunos textos aluden a la clasificación del proceso de abandono de acuerdo con el tipo de baja del sistema administrativo, a saber, si fue voluntario, forzoso, por normatividad, etc. No obstante, el alumno inscrito (y en cierta medida el que deserta) pierde relevancia y se convierte en un dato inanimado del proceso educativo (Muñoz et al., 2005; González, 2006; Román, 2009; entre otros).
} 
referencia varios estudios con enfoques integrales realizados en la Universidad de Quintana Roo (UQRoo), ubicada en el Caribe mexicano.

Debo mencionar que este texto es una propuesta metodológica emanada de varios proyectos de investigación educativa realizados en la UQRoo entre el 2011 y el 2013. Dichos estudios fueron financiados por la Secretaría de Educación Pública a través del Programa Integral de Fortalecimiento Institucional (PIFI) y el Fondo para Elevar la Calidad de la Educación Superior (FECES), fondos federales mexicanos.

Resta comentar que he convenido en estructurar la exposición considerando dos ejes elementales: a) la discusión teórico-metodológica de la propuesta que consiste en el análisis de pertinencia del uso de la etnografía institucional como dispositivo metodológico para la identificación de áreas de oportunidad en la educación superior; y b) algunos resultados de su aplicación usando como referencia los estudios realizados en la Universidad con el objetivo de exteriorizar los alcances de esta colocación. Resta decir que la idea central de esta propuesta consiste, básicamente, en la identificación de una suerte de grado de congruencia entre lo que se dice y lo que se pone en práctica y que, inexorablemente, va construyendo una lógica del desarrollo universitario expresado en su experiencia escolar.

\section{DISCUSIÓN TEÓRICO-METODOLÓGICA}

La etnografía, como sucede con algunos términos de la investigación social y educativa, ha sido víctima de adjetivaciones que, con solo mencionarle en Internet, se detona una serie incansable de respuestas. Cabe mencionar que una de ellas, sin duda interesante, refiere a la aplicación de la etnografía en el área de la educación (Pulido, 2003; Bertely, 1994; Álvarez, 2008; Serra, 2004; entre otros textos en formato PDF y otros electrónicos). No obstante lo persuasivo de estas propuestas, el uso del método etnográfico para construir realidades escolares subyace a una estrategia metodológica de investigación social embrionaria en el área educativa. 
La calidad de incipiente no radica en el uso en sí del método, sino en los alcances del mismo. Tal como aseguran los autores de algunos textos sobre el tema, sus propósitos redundan en identificar aspectos problemáticos del comportamiento en la escuela a través del método etnográfico. Gabriel Noel, por ejemplo, se propone analizar -a través de la etnografía- "la conflictividad entre agentes y destinatarios del sistema escolar en escuelas públicas de nivel primario [...] de barrios populares urbanos [...] de Buenos Aires" (Noel, 2009: 32). Otros, han convenido en realizar una compilación de los tipos de etnografías con el propósito de recalcar la importancia de su uso en el entorno escolar (Piña, 1997: 3-4; Álvarez, 2008). Otro ejemplo interesante es el de Guadalupe Balderas y Dionisia Ramírez, quienes otorgan un panorama revelador de los aportes de la etnografía como método integral en el estudio del comportamiento en la escuela. Para las autoras, el uso de la etnografía educativa (como le llaman) tiene el objetivo de "aportar valiosos datos de los contextos, actividades y creencias de los participantes en los escenarios educativos [que] corresponden a los procesos educativos tal como estos ocurren naturalmente" (Balderas y Ramírez, 2004: 77).

Sin duda, los aportes de la etnografía en la investigación educativa son mencionados por una cantidad impresionante de seguidores, en particular, partidarios de enfoques cualitativos. Sin embargo, la esencia de la versión romántica de este método -fundamentalmente descriptivo- no dirige hacia una discusión sobre sus posibilidades; y la adjetivación que se realiza de esta (como educativa) es respaldada por el contexto en el que se elabora. Es así que la propuesta sobre el rescate de la etnografía institucional problematizando el entorno escolar a la luz de los estatutos externos y, concretamente, en el sentido inverso, lleva implícito el encargo de ir más allá de una mera adjetivación del término (tal como supongo en el título de este apartado). Pero antes de proseguir sobre el tema, debo dejar clara mi concepción sobre el término etnografía.

La etnografía es un método holístico. Forma parte de un proceso amplio de investigación que solo puede entenderse desde su relación con el resto de sus partes; es decir, la etnografía no es tal, si no se compone del discurso, el imaginario, el comportamiento 
y la historia de quienes habitan en ella. Dicho de otro modo, esta es inocua si es mera descripción comportamental de una sociedad y de su entorno físico. También resulta inútil si no integra creencias y reglas sociales formales y consuetudinarias. Por reiterativo que parezca, entenderle como un mero proceso de descripción es subestimar los alcances de un método de análisis del material empírico adquirido en campo.

Ha sido recurrente la imagen literal de "descripción de los pueblos" como etimología de la palabra vinculada al estudio de los grupos indígenas o nativos. En antropología social, se ha intentado alejar de manera estricta y rigurosa los aspectos obligatorios de la investigación social. Me refiero a la visión etic y emic, esto es, una voz de redacción, "objetiva”, y otra que incluye el análisis del investigador y, si es necesaria, su implicación en el estudio. En el área de conocimiento mencionado, es común que se hagan ensayar diarios de campo separando los datos absolutamente vírgenes a nuestras opiniones de aquellas reflexiones, sensaciones y sentimientos (en caso de haberlos).

El método etnográfico debe ir más allá de la descripción en bruto basado, particularmente, en la observación, y recurrir a la integración no solo de otras herramientas, sino además de reflexiones surgidas en trabajo de campo y durante la cautelosa delineación de los detalles de una sociedad o grupo escolar. En lo específico, me refiero a una etnografía en su versión integral que debe tomarse como una especie de descripción reflexiva de la realidad en su conjunto. Es el resultado no solo de la observación, sino también de la conversación, de la fotografía, de la encuesta, de la bibliografía y contenido documental, y -vale mencionarde la subjetividad del investigador. En una frase, es la descripción problematizada.

No existe duda de la complejidad del método, de tal forma que la propia etimología de la palabra es insuficiente para explicar su concepción, al menos no en el sentido aquí expuesto. Ahora, cabe incursionar en los detalles de la etnografía institucional y decir en qué consiste, cómo aplicarla y en qué medida utilizarla. Debo decir que la incursión de este tipo de etnografía ha sido inusual en el área de la educación y su aplicación se ha limitado al análisis de la congruencia de ciertas políticas públicas dirigidas 
a la educación; pero en mayor medida, su aplicación se limita a la exploración del entorno laboral en instituciones gubernamentales a través de la identificación de problemáticas internas. En lo que sigue expondré algunos ejemplos del uso, para después aterrizar en una propuesta en el contexto de las instituciones de educación superior.

Ante todo, la etnografía institucional no dista mucho de otras etnografías planteadas en cualquiera de los enfoques cualitativos, esto es, la descripción sujeta a la realidad social estudiada. En un intento por deslindarse de la etnografía clásica (descripción densa de Clifford Geertz o la realizada por Bronislaw Malinowski), James Clifford (1991; mencionado en Pineda et al., 2006) plantea la posibilidad de una etnografía moderna; a partir de esta propuesta, fundamentada en el tema de la autoridad etnográfica y del análisis de los discursos, surge la propuesta para entender a la etnografía institucional como:

... la metodología que contextualiza y analiza los discursos, dentro de los cuales se establecen las relaciones y negociaciones entre al menos dos y habitualmente más sujetos (instituciones), conscientes y políticamente significantes en las intervenciones y entre los arreglos institucionales, para resolver los problemas socialmente construidos. (Pineda et al., 2006: 285).

Mencionado por los autores, uno de los primeros desarrolladores de la etnografía institucional fue James Ferguson (1990) y luego en colaboración con Larry Lohnmann (1994). En sus textos referentes al desarrollo y el poder burocrático en Lesoto, Sudáfrica, expone que la intervención de un proyecto de desarrollo no se basa en las necesidades reales del entorno social, sino -por el contrario-, la realidad social del medio da pie a la proliferación de empresas o agencias de desarrollo que al fin de cuentas influyen sobre la vida social y económica de la población en cuestión (Pineda et al., 2006: 285). Así, el acercamiento en este caso a través de la etnografía institucional admite ejes temáticos ligados a la gobernabilidad y a los alcances del proceso sobre la realidad social.

Ivonne Vizcarra usa de modo similar este método dirigiéndolo hacia el análisis de la institucionalización de la equidad de 
género. Entre sus conclusiones, comenta que la economía política feminista (generalmente entendida como el acceso al empleo de parte de las mujeres) debe conjuntar "un análisis de las intervenciones institucionales [...] y cómo estas intervenciones y arreglos pueden ser mejorados en beneficio, no solo de asegurar la reproducción del capital, sino de las propias mujeres y la sociedad en su conjunto" (Vizcarra, 2002: 91). Es en esta medida que el método integra desde la descripción ineludible hasta la colocación reflexiva del dato empírico.

Ya que la efectividad de la etnografía institucional en este documento recae en la exploración de la experiencia universitaria como categoría de análisis, conviene mencionar brevemente en qué consiste la experiencia en el campo de las ciencias sociales. Al respecto, vale decir que me refiero a la experiencia del universitario en el contexto de la institución, y hasta cierto punto, en la dimensión cotidiana. Así, la experiencia del universitario consiste en la manifestación de la subjetividad abstraída en la dimensión escolar; la interiorización de una serie de normas establecidas por medio de las relaciones escolares cotidianas en el proceso educativo. Por lo tanto, la experiencia universitaria debe entenderse como la concatenación de experiencias escolares previas: primaria, secundaria y bachillerato. Aunque en el aqui y ahora se ubiquen claramente en el discurso del estudiante.

El universitario está sujeto de experiencias. La manifestación de tales vivencias se reduce a formas de comportamiento. Henrik Ronsbo (2006) argumenta que las formas de manifestación de la experiencia no siempre suelen ser las más evidentes. Por lo tanto, él mismo considera a la experiencia como enigma, como una situación inenarrable como efecto entre la persona y la situación. De este modo, plantea que el problema no está en definir ni en aprehender la experiencia como tal, dado que es una tarea insostenible; para él, el problema reside en las formas de desplazamiento de esas experiencias en acciones discursivas o "performativas", individuales y colectivas. Es posible que no exista forma alguna de significación que dé cuenta en la experiencia, pero sí de significadores (palabras, actitudes, prácticas) que pueden hacer a esta asequible, descifrable y analizable. En tal caso, la reprobación (si la usamos como ejemplo) no es más que el síntoma de la expe- 
riencia escolar. Así, lo importante es desviar la mirada del hecho en sí y dirigirlo hacia el análisis del comportamiento escolar y del discurso universitario sin preguntarle de antemano ¿por qué reprobaste? Ya que muchas veces los fenómenos escolares como la reprobación o la deserción admiten discursos preelaborados del estudiante.

La experiencia, entonces, se convierte en un sitio de debate entre el pasado y sus diversas manifestaciones en el presente, al igual que sus proyecciones sobre el futuro en expectativas académicas, laborales o de desarrollo profesional. La experiencia es así subjetiva; la comunicación de esta es autoreferencial; es decir, se emite a través de la percepción del sujeto en primera instancia (Bruner, 1986: 5). Lo que se desea, entonces, es abstraer la reflexión de parte del universitario sobre sus vivencias en torno a su permanencia en la escuela. En esta dirección, cabe mencionar elementos de consideración de la experiencia escolar como: la edad, el sexo, el origen social, las prácticas cotidianas relacionadas con el estudio, la relación con los pares y con sus profesores, la vida escolar académica y no académica (o prácticas de consumo cultural dentro y fuera de la Universidad, por ejemplo), entre otras dinámicas (Guzmán, 2002). Entonces, es elemental rescatar ideas sobre la socialización y la individuación de los universitarios. Cabe advertir que la socialización debe entenderse como un proceso de construcción de experiencias, es decir, de interacciones que se desarrollan a través de un trabajo continuo del sujeto de aprendizaje (Dubet, 1994).

¿Cuál es la relevancia de la etnografía institucional en la exploración de la experiencia escolar universitaria? Los ejes analíticos a explorar en las experiencias escolares pueden estar circunscritas al mero entorno institucional, pero considerando el entorno social y el bagaje cultural del estudiante. Algunos ejes considerados al momento en los estudios realizados en la Universidad de Quintana Roo han sido: el entorno material, las relaciones inter e intragenéricas, inter e intrageneracionales (considerando la cohorte como la división generacional existente, por arbitraria que pueda parecer esta clasificación); además, las relaciones académicas y amistosas con los profesores y con los pares, la percepción sobre el plan de estudios y las asignaturas, y aquella 
sobre del éxito escolar propio. Por medio de su articulación con el "deber ser" universitario, esto es, la puesta en marcha (en mayor o menor medida) del plan de estudios (enfoque curricular, competencias docentes, entre otros modelos de aprendizaje), los reglamentos administrativos y otro tipo de reglas con las que, aunque no fundamentadas documentalmente, el estudiante va construyendo una visión sobre la vida en la universidad.

Sea de jure o de facto para uno y otros casos, lo cierto es que existe relación implícita entre -por decirlo de algún modo- lo que se dice, se hace y se piensa. Vale aclarar que la relación no siempre es positiva o bien directa. El propósito en la siguiente sección es identificar el tipo de relación existente entre el discurso institucional y la realidad estudiantil que dan sentido a la experiencia (o experiencias) de los universitarios. Aquí ubico la relevancia de realizar una tipo de etnografía reflexiva (problematizada) que al momento el término adecuado es el que se sugiere en el título.

¿Hacia dónde mirar cuando hacemos este ejercicio? Resulta importante plantear un punto de partida de acuerdo con nuestros objetivos o intereses de investigación. Cabe comentar que no se trata de integrar la mayor cantidad de aspectos en la etnografía institucional; incluso puedo asegurar que la definición de aspectos o dimensiones de este método recae en un primer acercamiento a las experiencias de los universitarios. Valdría preguntarnos entonces qué emerge de sus discursos y formas de comportamiento escolar para hacer enlaces con los aspectos institucionales a considerar en la etnografía. Al respecto, en la figura 1 -datos obtenidos a través de acercamientos cualitativos de observación y entrevistas colectivas (grupos de discusión) - muestro solo algunos de los aspectos a considerar en las etnografías institucionales en la investigación educativa; considero posible integrar muchas otras, como las relaciones de género, el acoso escolar (bullying) y bagaje cultural, por ejemplo. Ahora, el propósito fundamental es encontrar -a través de este ejercicio- los vínculos entre aspectos que den cuenta de una lógica institucional, esto es, un esquema básico que muestre una suerte de status quo del sistema educativo interno. Solo a través de la definición de este esquema es posible identificar sus debilidades y convertirlo -en el mediano y largo plazo- a 
uno de mayor integración positiva de sus aspectos. Dicho con un ejemplo, en el Modelo Educativo puede encontrarse un "deber ser" universitario ideal que no necesariamente encontramos en la vida escolar vigente, en el que el "ser" (o "seres") universitario es producto de su desarrollo escolar previo y actual; puede plantarse la pregunta sobre qué otros aspectos (y de qué manera) están implicados en el "ser". Encuentro que todos aquellos aspectos relacionados con el proceso educativo (práctica docente, las instancias académicas y el modelo curricular) y las relaciones intersubjetivas (académicas y amistosas con los pares y profesores) se involucran con este "ser" o conjunto de "seres".

FIGURA 1. Algunos aspectos del ambiente institucional a considerar en la etnografía

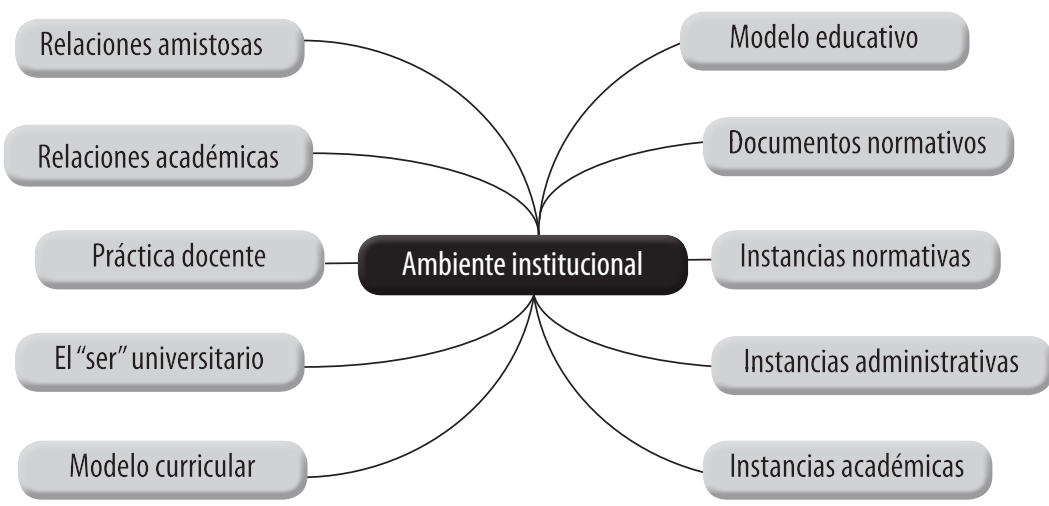

En efecto, la etnografía institucional -si se le toma en serio- es extensa y metodológicamente compleja, al menos en la investigación en educación. No obstante, los resultados son reveladores ya que se logra descubrir un modus vivendi de la educación como un microsistema educativo que opera casi por sí sola bajo un esquema coherente o no con el sistema educativo mayor, al que también convendría problematizar. A continuación muestro solo algunos resultados obtenidos de la aplicación de la etnografía institucional ya que el trabajo en extenso consta de numerosas cuartillas que, por cuestiones de espacio me he dado a la tarea de sintetizar. 


\section{ALGUNOS RESULTADOS}

En verano de 2011 elaboramos, con la colaboración de los miembros del Departamento de Innovación Educativa, el primer estudio sobre trayectoria escolar que involucró la aplicación de un instrumento dirigido a los estudiantes de nuevo ingreso. La propuesta era interesante dado que identificamos, en una de los campus, que más de $90 \%$ de los estudiantes encuestados estaban totalmente entusiasmados en cursar el primer semestre, independientemente de que haya elegido o no su carrera de interés. Sin duda era necesario un instrumento de seguimiento para conocer literalmente qué había ocurrido con estos universitarios luego de su primer semestre. Entonces, los estudios de trayectoria escolar estaban fundamentados en una metodología cuantitativa; pero hacía falta la integración de un acercamiento que diera cuenta no solo del indicio, sino además de la problemática estudiantil y, ante todo, de la percepción del estudiante con respecto a su vida en la universidad.

A través de grupos de discusión realizados con estudiantes de distintas carreras y semestres exploramos la opinión de los alumnos de manera libre, incluso podría decir arbitraria; lo anterior con el objetivo de que ellos mismos construyan -de acuerdo con su interés- las dimensiones de conversación y discusión. Dicho sea de paso, los grupos de discusión consistían en el planteamiento de situaciones problemáticas a los estudiantes con respecto a su vida escolar para hacer emerger dudas, cuestionamientos y discusiones que llevaran a la generación de reflexiones sobre su papel como universitarios; esto, a su vez, acarrea propuestas de su parte para la mejora educativa, más allá de simples quejas o demandas sin sustento. Debo comentar que realicé, con el apoyo de dos jóvenes universitarios, sesiones colectivas de entrevista que decidí llamar Grupos de discusión, a partir de lo establecido por Dubet y Martucelli:

Como la experiencia más individual permanece socialmente construida en el juego de relaciones con otras y con vinculaciones sociales, debe ser captada a través de la actividad de un grupo que testimonia una condición común y socialmente situada. [...] hay que captar la experiencia a través 
de un grupo para que emerja la especificidad de los recorridos y de las sensibilidades personales, evitando siempre que el testimonio se cierre sobre sí mismo, arrastrado a veces por la relación cara a cara de la entrevista. (Dubet y Martucelli, 1998: 16)

De esta manera, lo expuesto a continuación es la integración del discurso institucional, estudios institucionales elaborados con un enfoque cuantitativo y el discurso universitario emanado de los grupos de discusión, un acercamiento elementalmente cualitativo. Debo comentar que se ha mantenido el anonimato de los participantes para asegurar su confidencialidad. En lo que respecta a los documentos rectores de la Universidad usados en este escrito, estos se pueden consultar, de manera libre, en la siguiente liga: http://www.uqroo.mx/nuestra-universidad/documentos.

Atribuir de antemano al estudiante un papel de responsabilidad cuando ingresa por primera vez a la universidad es considerar que su formación previa no existe o bien no tiene relevancia alguna. Es normal, hasta cierto punto, si los profesores ven con sospecha a los nuevos universitarios que, por primera vez, se desprenden de un esquema de aprendizaje casi tutelar en el que fueron llevados de la mano en su egreso del nivel medio superior. Los mismos universitarios se percatan de la enorme brecha que existe entre lo que se espera de ellos en la universidad y lo que, de hecho, realizaban en la preparatoria (medio superior). En este sentido, el supuesto aprendizaje autónomo que muchas instituciones argumentan como posibilidad en los modelos educativos actuales es un verdadero reto dado que, al ingreso, los estudiantes no cuentan con las herramientas para serlo, al menos no en su totalidad (Vázquez, 2012, 131). Es así que la formación previa adquiere relevancia en la posible determinación del devenir estudiantil.

Si te gusta, no lo vas a sentir tanto, pero también hay compañeros que no están acostumbrados a leer, a esa cultura de la lectura; entonces, yo creo que ese es un problema que ya viene desde antes de ingresar a la carrera, porque yo me acuerdo que, cuando estudiaba en el bachillerato, solo leí dos que tres libros, pero solamente porque me obligo el bachillerato porque por otra parte... y no habría leído, siempre sentía: “ay es que ya 
me cansé de estar leyendo, ya me cansó Max Weber, ya me cansó, otro autor..." (Colaborador 3, GVDPLY-12).

En las Políticas Operativas de la UQRoo se asegura que los planes de estudio "incluirán un semestre cero que permita a los aspirantes ingresar o mejorar su desempeńo académico para continuar sus estudios superiores" (UQRoo, 2010b: 4). Es una suerte de estrategia de nivelación académica para aquellos universitarios con ciertas deficiencias en su formación previa. Sin embargo, ¿es posible nivelar un proceso educativo que no solamente involucra uno de los niveles de escolaridad? Aunque las intenciones sean buenas, la estrategia debe ser cautelosa en muchos sentidos, ya que aunque la propuesta esté ya normada en el documento institucional, queda la pregunta: ¿en qué medida se puede en un solo semestre desarrollar el razonamiento lógico-matemático o habilidades de lectoescritura?, planteado con base en el fragmento: "yo me acuerdo que, cuando estudiaba (...) solo leí dos que tres libros". 3

La formación previa es fundamental en la vida universitaria, no únicamente porque implica ventajas y desventajas al momento de adaptarse académicamente, en tanto se adquieren ciertas responsabilidades, sino además porque la trayectoria del estudiante en el medio superior resuelve muchas veces el devenir del universitario. Estos significa que las actitudes en su proceso de aprendizaje son reproducidas, sean estas positivas o negativas. Algunas instituciones ${ }^{4}$ analizan este proceso de modo cuantitativo al construir indicadores de trayectoria escolar, donde se puede visualizar la probabilidad de eficiencia terminal por medio de tres variables: la calificación final del bachillerato, la calificación del

\footnotetext{
${ }^{2}$ La clave de entrevista corresponde a mis iniciales y al campus en el que se realizó la entrevista, a saber: CHE-Chetumal, PLY-Playa del Carmen y COZ-Cozumel.

${ }^{3}$ Lo mismo ocurre con la redacción (acentuación, puntuación, construcción de enunciados, etc.). En mi experiencia docente, me he encontrado con serias deficiencias en la comunicación escrita de la mayoría de los estudiantes de nuevo ingreso (incluso de semestres avanzados) ¿Qué ocurre con las habilidades de lectoescritura? Escribimos desde la primaria, lo hacemos en la secundaria y, sin duda, lo realizamos en el bachillerato ¿Es posible recuperar en un semestre la comunicación escrita apropiada, así como el "gusto" por la lectura?

${ }^{4}$ La Universidad Veracruzana (UV), México, por medio del trabajo continuo de Ragueb Chaín han desarrollado sistemas de identificación de las trayectorias escolares de modo eficiente. No obstante, el hecho de ser cerrados en demasía, no los hace aplicables en otras instituciones en las que se consideran otros factores implícitos en el comportamiento escolar.
} 
examen de admisión (en este caso EXAN-II del Centro Nacional de Evaluación) y de la forma de acreditación de las asignaturas en el medio superior. Si bien la propuesta es interesante, el ejercicio no rindió muchos frutos para la UQRoo por ser de un contexto y sistema diferentes. Ante esta propuesta, elaboré -con la colaboración de personal de la Universidad- un instrumento con el fin de identificar perfiles de ingreso. Así, se obtuvo información que puso en evidencia el tipo de estudiantes que se recibieron en 2011, 2012 y 2013 en la Institución (véase figura 2).

Entre otros aspectos, solicitamos a los aspirantes a universitarios valorar del 1 al 5 la frecuencia con que, durante el bachillerato, realizaban ciertas actividades escolares comunes en la vida de un estudiante. Por medio de la media armónica se representaron las respuestas por división académica. El hallazgo fue que la Universidad de Quintana Roo recibe estudiantes con un perfil pasivo en estas tareas; es decir, es más frecuente que los estudiantes tomen apuntes y lleguen puntuales a clases. Al contrario, la preparación de exposiciones, participaciones y el trabajo en equipo (actividades que pueden considerarse como comunes en la educación superior) son menos frecuentes. Es también relevante mencionar que, en todas las divisiones académicas, la tendencia es la misma.

FIGURA 2. Gráfica de las actitudes escolares de estudiantes de nuevo ingreso 2012

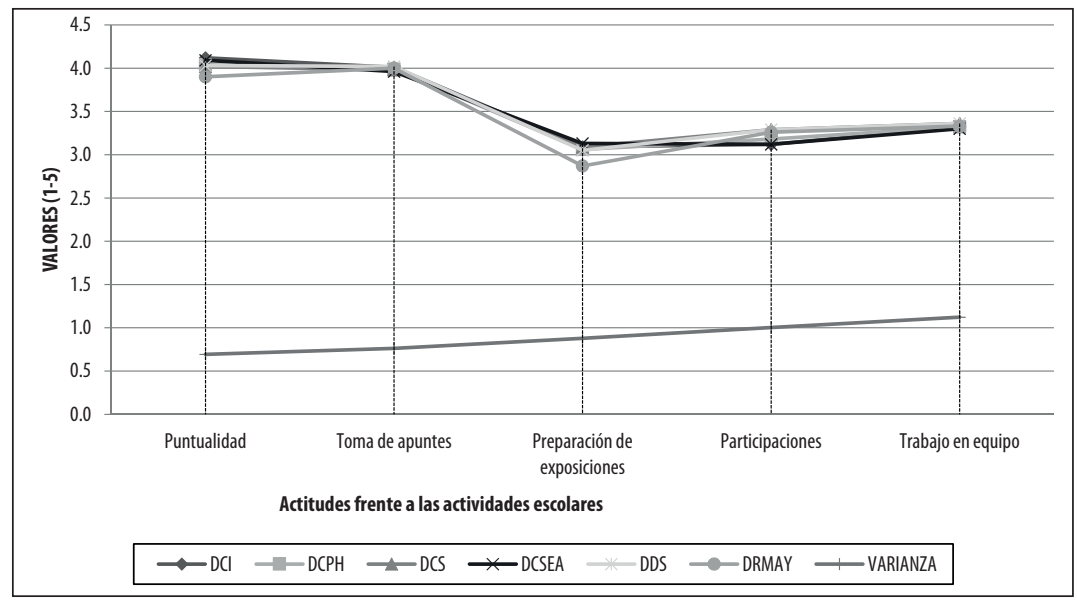


Este hallazgo lleva a problematizar lo que en líneas anteriores mencioné de modo superficial: aprendizaje autónomo. Al respecto, la noción más elemental es que el proceso educativo se base en la forma con la que el estudiante aprende, y menos en la manera como se enseña. En este sentido, el papel del profesor se limita a la coordinación de las actividades dirigidas al aprendizaje. En esta versión, es el universitario quien plantea sus propias posibilidades de acuerdo con sus intereses y su vocación; por su parte, la universidad es la responsable de establecer los mecanismos apropiados para que dicho proceso se ejecute de modo óptimo.

El aprender autónomo implica responsabilizarse de la propia formación, aprender por cuenta propia mediante el estudio personal, a partir de la propia comprensión, pero sobre todo, mediante la acumulación de experiencias ajenas comunicadas por múltiples medios. El aprender autónomo compromete al estudiante a responsabilizarse de su propio desarrollo académico, con el respaldo del profesor y de las áreas de atención y servicios estudiantiles, para lograr su formación integral (UQRoo, 2010a: 13-14).

La propuesta es tentadora. Pensar en un escenario donde el joven universitario pueda construir su propio camino de aprendizaje basado en su experiencia y en los medios provistos por la institución es, por demás, el sueño de todo educador. La visión inmersa en el aprendizaje autónomo es una que merece considerarse por cuanto -como propuesta- implica el desarrollo fundamental de todo ser humano, esto es, ser autoconsciente de su aprendizaje. Deberemos pensar si los jóvenes que egresan del nivel medio superior, ¿están listos para tomar tal responsabilidad? Lo cierto es que tanto profesores como los funcionarios responsables del modelo educativo de cada institución deben afrontar este reto de modo estratégico, es decir, tomar en cuenta el contexto y el perfil de los estudiantes que reciben para lograr desarrollar la actitud educativa apropiada. Pero también es verdad que los estudiantes no son indiferentes a este proceso y en la mayoría de los casos dan cuenta de sus necesidades y áreas de oportunidad.

Otro aspecto relativo a la integración del estudiante al modelo curricular se vincula con la situación laboral. En otoño de 2013, la encuesta de "Ingreso y experiencia escolar previa" arrojó 
datos interesantes sobre la situación laboral: jornada de trabajo, relación con la carrera de elección y la posibilidad de abandono (véase cuadro 1; 23\% de la muestra admitió tener algún tipo de empleo; esto es relevante en la integración de los estudiantes que trabajan con un modelo educativo en el que se privilegia, según los documentos rectores, el aprendizaje.

CUADRO 1. Situación laboral [estudiantes de nuevo ingreso 2013]

\begin{tabular}{|c|c|c|c|}
\hline Variable & Estudiantes co & & Totales \\
\hline \multirow{3}{*}{ Jornada laboral } & Tiempo completo & 33 & \multirow{3}{*}{140} \\
\hline & Medio tiempo & 65 & \\
\hline & Horario variado & 42 & \\
\hline \multirow{3}{*}{ Relación del empleo con la carrera en curso } & Mucha relación & 33 & \multirow{3}{*}{$\begin{array}{c}137 \\
\text { (3 perdidos) }\end{array}$} \\
\hline & Algo de relación & 39 & \\
\hline & No se relaciona & 65 & \\
\hline \multirow{2}{*}{ Abandono por prioridad laboral } & Sí abandonaría & 18 & \multirow{2}{*}{$\begin{array}{c}135 \\
\text { (5 perdidos) }\end{array}$} \\
\hline & No abandonaría & 117 & \\
\hline
\end{tabular}

El Modelo Educativo y las Políticas Operativas de la Institución en cuestión vislumbran un concepto fundamental: la flexibilidad curricular. Como en el caso del aprendizaje autónomo, en la práctica esta noción debe tomarse con cautela. La flexibilidad curricular implica que cualquier universitario de cualquier programa educativo y semestre pueda matricularse en las materias consideradas generales lo que conlleva, en la mayoría de los casos, a la creación de grupos mixtos. Una de las desventajas prácticas de esta versión de flexibilidad es la dificultad para dirigir temáticamente la asignatura general. Dicho de otro modo, algunos profesores no logran realizar un vínculo claro entre la materia general (matemáticas, inglés, redacción, comunicación oral, etc.) y su relevancia en el plan de estudios debido a que alumnos de diferentes carreras cursan tal asignatura ${ }^{5}$. En la mayoría de los ca-

\footnotetext{
${ }^{5}$ En un estudio realizado en 2013 para identificar posibles factores que orillan a la reprobación y deserción, uno de los aspectos más mencionados fue (en el caso de la reprobación) encontrar las asignaturas irrelevantes (un subgrupo fue "asignaturas difíciles").
} 
sos, la flexibilidad curricular está condicionada con la posibilidad de apertura de una asignatura general. Como en el siguiente fragmento, la flexibilidad es limitada y puede culminar en el rezago de más de un estudiante.

(...) sí, es que un problema que hay es que las materias no se abren y te vas atrasando, y tiene que tomar en cuenta si no viene corriendo tras la materia para que se abra, lo que más atrasa no es que repruebes, lo que más atrasa es que las materias no se abran, ¿por qué?, porque solo dos personas lo van a llevar, pero solo dos personas estamos inscritas, cómo quieren que le hagamos (Colaborador 8, GVDCHE-5).

En casos en los que los estudiantes cuentan con trabajo remunerado la realidad es distinta. El tema adquiere un tono importante dado que quienes trabajan ven con buenos ojos la posibilidad de matricularse en un número de materias distinto y en horarios que más les convengan, siempre y cuando estén disponibles. Si bien el debate en torno a la flexibildiad curricular puede ser interminable, en especial si se consideran los diferentes puntos de vista, lo cierto es que los estudiantes están conscientes de su papel en la universidad y conocen sus deficiencias. Lo importante es tomar estas áreas de oportunidad, volcarlas en estrategias reales efectuadas en el aula.

(...) tener un sistema semiescolarizado y dándole la apertura para esas personas que sí quieren continuar sus estudios profesionales, que se quieren capacitar; además, esas personas tienen ya la experiencia laboral, imagínate a una persona que tiene la experiencia laboral más los estudios, yo creo que se va a complementar mucho y van hacer útiles en la sociedad que estén preparados y pues, obviamente, impulsas ese, este... cómo se llama (...) ya no vamos a estar rezagados en cuanto a nivel superior; entonces, yo sí conozco a muchas personas que me dicen yo no sigo estudiando porque, porque, pues, no tengo tiempo, porque mi trabajo es súper demandante (...) (Colaborador 6, GVDPLY-1).

El contar con un empleo como el anterior, puede tener un efecto positivo en el desempeño del universitario. Esto significa que los universitarios tienen la posibilidad de poner en práctica lo 
aprendido en clases, o bien, reforzar la participación constante en el aula con la experiencia laboral. Sin duda el tiempo disponible para el estudio puede ser un talón de Aquiles al que la flexibilidad deberá dar respuesta oportuna, dado que una cantidad importante de estudiantes tienen la necesidad de costear sus estudios por medio del trabajo remunerado, incluso si la labor que desempeñan no se relaciona en absoluto con la carrera que estudian. (Véase cuadro 2).

CUADR0 2. Situación laboral [Muestra general 2012] ${ }^{6}$

\begin{tabular}{|l|c|c|c|}
\hline \multirow{2}{*}{ Variable } & \multicolumn{2}{|c|}{ Estudiantes con empleo } & \multirow{2}{*}{ Totales } \\
\hline \multirow{2}{*}{ Jornada laboral } & Tiempo completo & 103 & \multirow{2}{*}{255} \\
\cline { 2 - 3 } & Medio tiempo & 152 & \\
\hline \multirow{3}{*}{ Relación del empleo con la carrera en curso } & Mucha relación & 76 & \multirow{2}{*}{255} \\
\cline { 2 - 3 } & Algo de relación & 78 & \multirow{2}{*}{255} \\
\cline { 2 - 3 } & No se relaciona & 101 & \\
\hline \multirow{2}{*}{ Abandono por prioridad laboral } & Sí abandonaría & 70 & \multirow{2}{*}{255} \\
\cline { 2 - 3 } & No abandonaría & 185 & \\
\hline
\end{tabular}

Es un verdadero desafío para la institución, específicamente, para los responsables de las áreas de innovación educativa y aquellas de apoyo estudiantil, incluyendo a los profesores y a las áreas administrativas configurar un esquema de flexibilidad incluyente; en particular, cuando notamos que 70 alumnos en 2012 y 18 en 2013 pudieron haber abandonado sus estudios por prioridades laborales.

Es importante mencionar que los responsables de las áreas de apoyo académico hacen todo lo posible por resarcir las deficiencias institucionales en términos académicos. Incluso, en un documento estratégico titulado Plan de Implementación del Modelo Educativo, existe un apartado sobre la forma en la que se planean acciones concretas sobre la puesta en marchar del Modelo Curricular (en el que se plantea la flexibilidad curricular) por medio del rediseño curricular de los planes de estudio y de la Formación

\footnotetext{
${ }^{6}$ La opción "Horario variado" se anexó en 2013 a la encuesta de ingreso. Ese año no se realizó encuesta de seguimiento, razón por la cual muestro los resultados del año anterior.
} 
Docente por medio de la actualización didáctica (UQRoo, 2012: 49-50). Es importante señalar que aunque los estudiantes no sean expertos en pedagogía, saben que sus planes de estudio requieren mejoras, como lo indica la explicación elocuente de una estudiante de cuarto semestre:

(...) aplicar aún más como esos conocimientos con más actividades que nos ayudaran a mostrar o a desarrollar los conocimientos que vamos adquiriendo en algo ya más real; en nuestro caso, por ejemplo, ahorita estamos viendo una materia que se llama Cadenas productivas y dentro del plan está que vamos a elaborar algunos proyectos, y sé que todas las materias están encadenadas y se llevan unas con otras; entonces, me gustaría como que pudiéramos integrar un poquito desde el principio esas actividades que nos van a ayudar a integrar cada una de las materias en nuestras carreras porque, tal vez, de repente se están viendo las materias un poquito aisladas, como matemáticas y "no me metas con estadística y no me combines a mí cadenas productivas con esto", cuando, si se combinan... entonces, siento que debemos hacer algo que pudiera integrar todas esas materias como para que podamos estar viendo más de cerca la aplicación y, en el momento en el que estamos aprendiendo, estamos comprendiendo y viendo la aplicación en una situación real (Colaborador 8, GVDPLY-2).

Aunque existen muchos temas por los cuales problematizar la realidad escolar con respecto a la propuesta institucional, me he limitado en analizar y debatir el aprendizaje autónomo y la flexibilidad curricular establecida en los documentos rectores de la Universidad, a la luz de la vida escolar universitaria. El discurso universitario revela las áreas que merecen mayor atención y que, indudablemente, sostienen efectos sobre la reprobación y la deserción.

Dicho lo anterior, la etnografía institucional repara en la posibilidad de contrastar entre lo que se dice (en este caso documentos institucionales), con la experiencia escolar; resuelve aspectos como: ¿cómo viven los estudiantes su carrera? ¿Cuáles son los mecanismos que las instituciones configuran para implementar su discurso general? Además, integré datos que otorgan evidencia complementaria de la realidad escolar. Mientras más elementos 
tengamos para colocar al frente de la discusión, mayor consistencia tendrá nuestra "descripción” del ambiente institucional.

\section{CONCLUSIONES}

Los problemas más comunes referentes a la permanencia en la educación superior en México pueden tener explicaciones alternas a la pobreza y la marginación en la que vive un sector importante del país. Con lo anterior no quiero obviar la relación del rezago educativo con el poder adquisitivo de muchas poblaciones mexicanas. Sin embargo, la reprobación y deserción son problemáticas que se asocian a una serie de aspectos en los que encajan otro tipo de influencias. Por este motivo es fundamental optar por metodologías alternativas que integren acercamientos novedosos para lograr demarcar ejes de acción de mejora. Así, la etnografía institucional nos ayuda a desenmascarar la congruencia entre las propuestas y las acciones.

La problematización de los documentos institucionales, el contraste con los estudios cuantitativos realizados en un periodo de tres años y los grupos de discusión realizados (acercamiento cualitativo) son un punto de partida para la etnografía institucional, la cual implica mucho más que una mera descripción. Así, he explorado el uso de la etnografía como un método dirigido hacia la identificación de congruencia entre la propuesta educativa institucional y la experiencia estudiantil; aunque -sin lugar a dudas- queda mucha tarea pendiente.

Es nuestro deber abrir el discurso universitario en cada uno de sus ejes problemáticos y configurar estrategias para la mejora educativa en su nivel práctico. Por tal motivo, es importante atar cabos sueltos para que estas ideas compartidas lleven a la exploración de nuevos contextos y a la resolución de problemáticas de antaño. Resta decir que aunque la propuesta haya sido breve, los alcances son inconmensurables si se realiza de modo apropiado; por lo tanto, la etnografía institucional puede ser el mecanismo metodológico para conocer la medida en que el efecto real del proceso educativo -en este caso- en las instituciones de educación superior. 
LA ETNOGRAFÍA INSTITUCIONAL: UNA ALTERNATIVA METODOLÓGICA...

\section{REFERENCIAS BIBLIOGRÁFICAS}

Álvarez Álvarez, C., "La etnografía como modelo de investigación en educación", Gazeta de Antropología, vol. 24, núm. 1, 2008. Disponible en: http://hdl.handle.net/10481/6998

Balderas, G. y Ramírez, D., "Investigación etnográfica de la educación", en M. E. Mureta (Coord.), Alternativas metodológicas para la investigación educativa, México, AMAPSI Editorial/CESE, 2004, pp. 71-84.

Bertely Busquets, M. Retos metodológicos en etnografía de la educación, México, Universidad Veracruzana, 1994. Disponible en : http://www.uv.mx/cpue/coleccion/n_2526/pu blmari.htm. Fecha de Consulta, 20 de marzo de 2016.

Bruner, Edward M., "Experience and Its Expressions", en V. W. Turner y E. M. Bruner (Eds.) The Anthropology of Experience, Chicago, University Illinois Press, 1986, pp. 3-30.

Dubet, F., "Dimensions et figures de l'experience dans la université de masse", en Revue Francaise de Sociologie, vol. 35, núm. 4, 1994, pp. 511-532.

Dubet, F. y Martucelli, D., En la escuela. Sociología de la experiencia escolar, Buenos Aires, Losada, 1998, 489 pp.

Ferguson, J., The anti-politics machine: "Development", depoliticization and bureaucratic power in Lesotho, Cambridge, Cambridge University Press, 1990.

Ferguson, J. y Lohnmann, L., "The anti-politics machine: "Development" and bureaucratic power in Lesotho", en The Ecologist, vol. 24, núm. 5, 1994, pp. 176-181.

González González, M. T., "Absentismo y abandono escolar: una aproximación singular de la exclusión educativa”, en Revista Electrónica Iberoamericana sobre Calidad, Eficacia y Cambio en Educación, vol. 4, núm. 1, 2006, pp. 1-15.

Guzmán, C, "Reflexiones en torno a la condición estudiantil. Los aportes de la sociología francesa”, en Perfiles educativos, vol. 24, núm. 97-98, 2002, pp. 38-56.

Muñoz Izquierdo, C., Rodríguez, P. G. , Restrepo de Cepeda, P. y Borrani, C., "El síndrome del atraso escolar y el abandono del sistema educativo", en Revista Latinoamericana de Estudios Educativos, vol. 35, núm. 3-4, 2005, pp. 225-285. 
Noel, G., La conflictividad cotidiana en el escenario escolar: una perspectiva etnográfica, Argentina, UNSAM EDITA de Universidad Nacional de General San Martín, 2009, 234 pp.

Pineda Ruiz, S., Vizcarra Bordi, I. y Lutz Bachère, I., "Gobernabilidad y pobreza: proyectos indígenas mazahuas del Estado de México”, en Indiana, núm. 23, 2006, pp. 283-307.

Piña Osorio, J. M., "Consideraciones sobre la etnografía educativa”, en Perfiles Educativos, vol. 19, núm. 78, 1997. Disponible en, http://www.redalyc.org/articulo.oa?id=13207804. Fecha de consulta, 2 de febrero de 2016.

Pulido Moyano, R., "Una visión sobre la etnografía educativa a través del caso de unos alumnos del etnógrafo que sustituía a la maestra", en Ágora digital, núm. 6, 2003. Disponible en http://rabida.uhu.es/dspace/bitstream/handle /10272/3520/b15761794.pdf?sequence=1. Fecha de consulta, 15 de noviembre de 2015.

Román, M., "El fracaso escolar de los jóvenes en la enseñanza media. ¿Quiénes y por qué abandonan definitivamente el liceo en Chile", en Revista Electrónica Iberoamericana sobre Calidad, Eficacia y Cambio en Educación, vol. 7, núm. 4, 2009, pp. 95-119.

Ronsbo, H., "Displacing enigma and shaping communal hegemonyTowards the analysis of violent experience as social process", en Dialectical Anthropology, núm. 30, 2006, pp. 147-167.

Serra, C., "Etnografía escolar, etnografía de la educación", en Revista de Educación, núm. 334, 2004, pp. 165-176.

Universidad de Quintana Roo. Modelo educativo (Resumen ejecutivo). México: UQRoo, 2010a, 22 pp.

Universidad de Quintana Roo. Políticas operativas. México: UQRoo, 2010b, 15 pp.

Universidad de Quintana Roo. Plan de implementación del modelo educativo. México: UQRoo, 2012, 106 pp.

Vázquez Dzul, G., "El rizoma como fundamento para la elaboración de estrategias pedagógicas en la construcción de conocimiento y el diseño de programas de asignatura”, en Revista Educarnos, vol. 1, núm. 4, 2012, pp. 117-132.

Vizcarra Bordi, I., "La institucionalización de la equidad de género en el Estado de México y la economía política feminista”, en Convergencia, núm. 30, 2002, pp. 79-95. 